\title{
Distribuição espacial do índice de precipitação padronizada no Estado do Pará em
}

\section{uma década}

\author{
Spatial distribution of the standardized precipitation index in the State of Pará in a decade \\ Distribución espacial del índice de precipitación estandarizado en el Estado de Pará en una década
}

Recebido: 20/09/2021 | Revisado: 29/09/2021 | Aceito: 23/10/2021 | Publicado: 25/10/2021

\author{
Breno Eduardo dos Santos Alves \\ ORCID: https://orcid.org/0000-0003-4500-821X \\ Universidade Federal Rural da Amazônia, Brasil \\ E-mail: brenoedu_leao@hotmail.com \\ Alexandre Fernandes Santos Filho \\ ORCID: https://orcid.org/0000-0002-1684-3788 \\ Universidade Federal Rural da Amazônia, Brasil \\ E-mail: alexandresff25@gmail.com \\ Carlos Rodrigo Tanajura Caldeira \\ ORCID: https://orcid.org/0000-0001-7102-7735 \\ Universidade Federal Rural da Amazônia, Brasil \\ E-mail: caldeiracrt@gmail.com \\ Francimary da Silva Carneiro \\ ORCID: https://orcid.org/0000-0002-1693-8779 \\ Universidade Federal Rural da Amazônia, Brasil \\ E-mail: francimarycarneiro@gmail.com \\ Frank Bruno Baima de Sousa \\ ORCID: https://orcid.org/0000-0003-2548-0701 \\ Climatempo - A StormGeo Company, Brasil \\ E-mail: frank.sousa@climatempo.com.br \\ Glayson Francisco Bezerra das Chagas \\ ORCID: https://orcid.org/0000-0002-5796-1924 \\ Secretaria de Estado de Meio Ambiente e Sustentabilidade, Brasil \\ E-mail: chagasmeteorologista@gmail.com \\ Klewton Adriano Oliveira Pinheiro \\ ORCID: https://orcid.org/0000-0003-2696-4249 \\ Instituto Federal do Pará, Brasil \\ E-mail: klewton.pinheiro@gmail.com
}

\begin{abstract}
Resumo
A distribuição de chuvas é fundamental para o correto planejamento, a nível governamental, de estratégias contra desastres naturais, abastecimento de água, geração de energia e transporte hidroviário, e em nível de produção, por ser importante para gerenciar atividades que dependem do ciclo de chuvas. Objetivando distribuição de chuvas no periodo de dez anos em dois municipios do estado do Pará, fez-se a aplicação do Índice de Precipitação Padronizada, entre os anos de 2005 a 2015, em série semestral, para visualizar como está o comportamento de secas. Os dados foram obtidos na plataforma Giovanni, da NASA, e, após processados e plotados pela aplicação Panoply, foram espacializados no QGIS. Os resultados demonstraram uma concentração de umidade no sul e oeste do Estado de maneira mais evidente, o que foi corroborado pelos dados do Instituto Nacional de Meteorologia. Por fim, o uso do Índice de Precipitação Padronizado mostrou-se adequado para observações de ordem semestral de distribuição de umidade para o estado do Pará.
\end{abstract}

Palavras-chave: Sensoriamento remoto; Precipitação; Geoprocessamento.

\begin{abstract}
The distribution of rain is fundamental for the correct planning, at the governmental level, of strategies against natural disasters, water supply, energy generation and waterway transport, and at the production level, as it is important to manage activities that depend on the rain cycle. Aiming to know the reality of the state of Pará, the Standardized Rainfall Index was applied, between the years 2005 to 2015, in a semiannual series, to visualize the behavior of droughts. The data were obtained from the Giovanni platform, from NASA, and, after being processed and plotted by the Panoply application, they were spatialized in QGIS. The results showed a more evident concentration of moisture in the south and west of the state, which was corroborated by data from the National Institute of Meteorology. Finally, the use of the Standardized Precipitation Index proved to be adequate for semiannual observations of moisture distribution for Pará.
\end{abstract}

Keywords: Remote sensing; Precipitation; Geoprocessing. 


\begin{abstract}
Resumen
La distribución de la lluvia es fundamental para la correcta planificación, a nivel gubernamental, de estrategias contra desastres naturales, abastecimiento de agua, generación de energía y transporte fluvial, y a nivel de producción, ya que es importante gestionar las actividades que dependen del ciclo de la lluvia. . Con el objetivo de conocer la realidad del estado de Pará, se aplicó el Índice de Precipitaciones Estandarizado, entre los años 2005 a 2015, en una serie semestral, para visualizar el comportamiento de las sequías. Los datos se obtuvieron de la plataforma Giovanni, de la NASA y, luego de ser procesados y graficados por la aplicación Panoply, fueron espacializados en QGIS. Los resultados mostraron una concentración de humedad más evidente en el sur y oeste del estado, lo que fue corroborado por datos del Instituto Nacional de Meteorología. Finalmente, el uso del Índice de Precipitación Estandarizado resultó ser adecuado para las observaciones semestrales de la distribución de la humedad para Pará.
\end{abstract}

Palabras clave: Detección remota; Precipitación; Geoprocesamiento.

\title{
1. Introdução
}

O conhecimento acerca do clima e do comportamento da precipitação na esfera regional, estadual e municipal é de fundamental importância para os seus gestores, no que tange à gestão de riscos e abastecimento de água, e para pessoas ligadas a atividades que envolvam os ciclos de chuva, como agricultores, pescadores, marinha, aeronáutica, turismo e meio ambiente (Terassi et al., 2018, INMET, 2021). Dados do setor de Clima e Recursos Naturais da Organização das Nações Unidas para a Agricultura e Alimentação (FAO) apontam que, no mundo, mais de três bilhões de pessoas em áreas agrícolas de todo o mundo estão sofrendo com a baixa umidade do solo. E que, até 2050, a demanda global de água aumentará de $20 \%$ a $30 \%$ (Grilli, 2021). A disponibilidade de chuva em uma bacia durante um período de tempo é fator determinante para quantificar a necessidade de irrigação de culturas, o abastecimento de água doméstico e industrial, a geração de energia elétrica, O dimensionamento de drenos, vertedouros de barragens e obras contra cheias (Embrapa, 2004, Santos et al 2010). Além disso, a determinação da intensidade da precipitação é importante para o controle de cheias e da erosão do solo (Mol, 2005). Sendo o solo um recurso de renovação lenta e podendo ser rapidamente degradado pela ação da erosão, sendo fundamental o conhecimento da forma como a precipitação atua no ambiente no qual esteja inserido (Santos et al, 2010).

Uma observação meteorológica consiste na medição, registro ou determinação de todos os elementos que, em seu conjunto, representam as condições meteorológicas num dado momento e em determinado lugar, utilizando instrumentos adequados e valendo-se da vista (INMET, 2021). Os dados de chuva de uma determinada região são medidos a partir de pluviômetros ou pluviografos instalados em estações meteorológicas de superfície, distribuídas aleatoriamente no espaço (Vianello, 2011, Balbino, 2016). Porém o sensoriamento remoto vem sendo utilizado em registro da precipitação pluvial, o auxílio das imagens de satélites para elaborar a previsão em curto prazo de tempo e o radar meteorológico, que fornece as condições meteorológicas reinantes num espaço de tempo menor e também para uma área menor. O uso de satélites para monitorar o clima está ganhando espaço no cenário acadêmico e agrícola, já que a detecção remota é uma ferramenta útil, especialmente, em regiões com dados imprecisos ou mal controlado (Wagner et al., 2012). Uma dessas técnicas desenvolvidas foi a missão Tropical Rainfall Measuring Mission (TRMM) este satélite foi construído especificamente para monitorar e estudar a precipitação, principalmente nos trópicos e acompanhar a influência do clima em cenário global. Estimando valores de chuva a cada três horas no globo terrestre, é uma combinação de estimativas baseadas em imagens no infravermelho com microondas (Collischonn et al., 2016, Huffman et al., 2007; Zhong, 2012, Silva et al 2020).

O Standardized Precipitation Index (SPI) ou Índice Padronizado de Precipitação (IPP) é um indicador da pluviosidade acumulada ao longo de uma série temporal e tem por finalidade auxiliar no entendimento dos períodos de seca e abundância pluviométrica, sendo muito utilizado para estudos sobre implicações agrícolas, meteorológicas, hidrológicas e socioeconômicas do fenômeno seca com o objetivo de quantificar déficits de precipitação e como eles atuam sobre determinados segmentos, em diversas escalas de tempo (Siqueira \& Nery, 2017).

Diante do exposto, tivemos como objetivo identificar a distribuição acumulada de chuvas no período de dez anos, do 
ano de 2005 ao ano de 2015, em intervalos semestrais, com duas estações meteorológicas, nos municípios de Belém e de Itaituba, no Estado do Pará, na região Norte do Brasil.

\section{Metodologia}

\section{Área de Estudo}

O processamento se deu nos limites que englobam o estado do Pará $\left(-48.65^{\circ},-1.55^{\circ},-48.25^{\circ},-0.95^{\circ}\right)$ na região norte do Brasil (Figura 1), caracterizado, pela classificação climática de Köppen em trabalho elaborado por Alvares et. al. (2014), como majoritariamente Am (66\% do território), seguido por Af (28,4\%) e Aw (4,9\%), caracterizada pelo clima tropical (grupo A), com temperatura média de $18^{\circ} \mathrm{C}$ ou superior e precipitação significativa.

Figura 1 - Mapa de localização da área de estudo.
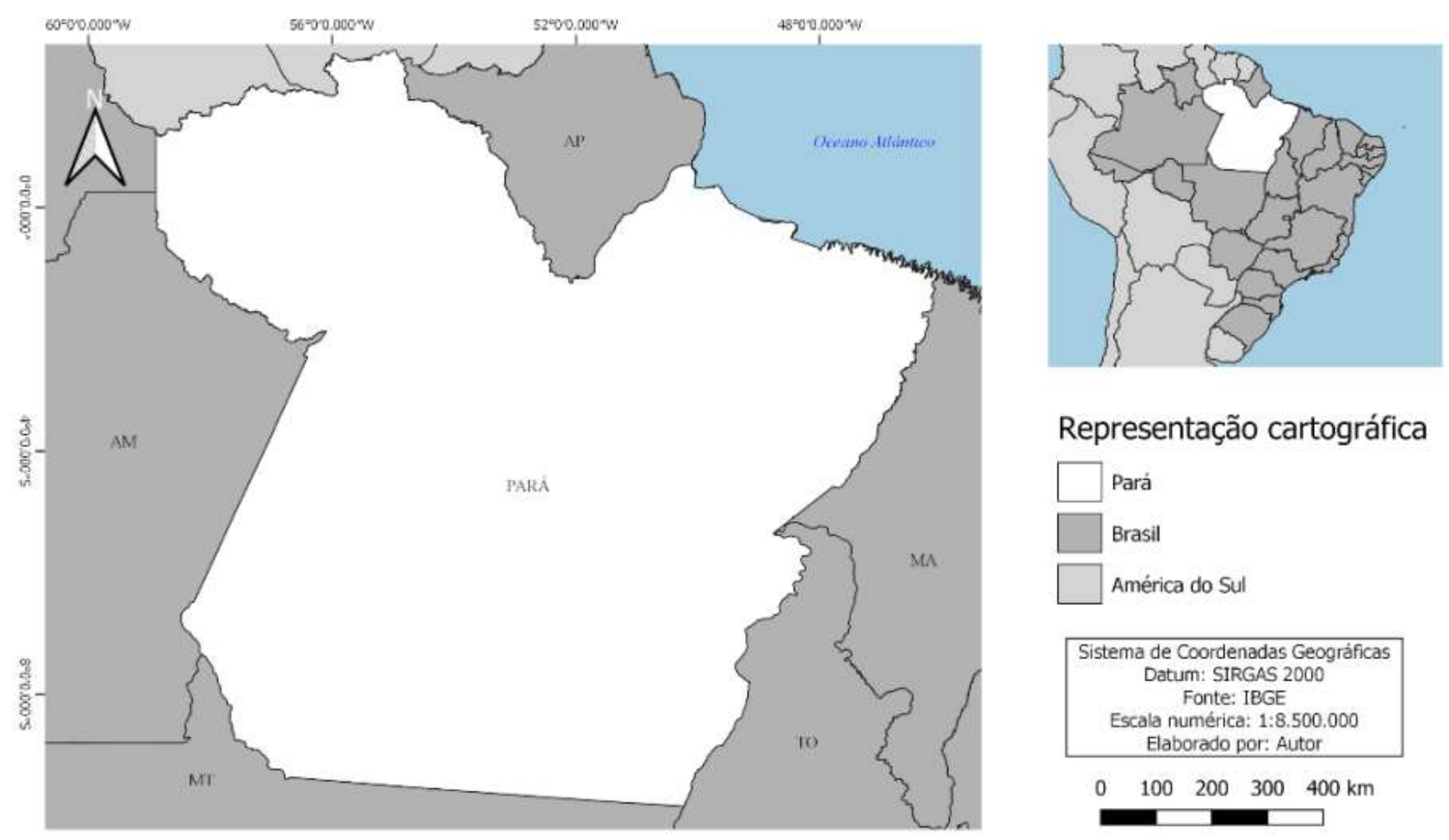

Fonte: Autores.

\section{Coleta dos Dados}

Os dados pluviométricos foram adquiridos na plataforma Giovanni, um serviço aberto da National Aeronautics and Space Administration (NASA) que provê informações abundantes de sensoriamento remoto de maneira simplificada sem necessariamente baixar os dados. Para este estudo, foram utilizados os dados de precipitação mensais de 2005 a 2015 por satélite denominada de Recuperações Multi-satellitE integradas para medida de precipitação global (sigla IMERG em inglês) que reúne uma constelação de satélites artificiais para o propósito de medições pluviométricas (NASA, 2020).

\section{Índice de Precipitação Padronizado - IPP}

Baseado somente na precipitação, é um índice usado para monitorar condições em uma determinada escala de tempo, neste caso, uma escala de 6 meses, que compara a precipitação desse período para o mesmo período ao longo dos anos. É um período eficaz para distinguir estações e até associar com níveis de reservatório (National Drought Mitigation Center, 2020). 
Valores de -1 e inferiores indicam condições de seca e valores de 1 e superiores indicam condições de umidade (ARSET, 2020).

\section{Processamento}

A página web do National Integrated Drought Information System (NIDIS) do Portal da Seca dos Estados Unidos fornece um software de código aberto desenvolvido na linguagem de programação de computador Python que recebe e processa os arquivos de precipitação calculando o SPI e levando em consideração também suas posições geográficas (NIDIS, 2020).

\section{Análise dos Dados}

A ferramenta Panoply disponibilizada pela ARSET plota os índices de acordo com as projeções definidas e valores calculados, além de exportar como imagem georreferenciada com seus valores em cada pixel, que posteriormente é utilizada para visualização no software QGIS

3.10. Além disso, foram comparados os resultados obtidos pelo IPP com os dados disponibilizados pelo Instituto Nacional de Meteorologia (INMET).

\section{Resultados e Discussão}

O resultado gera uma imagem georreferenciada com valores do índice em cada canal da imagem, como por exemplo, plotando um índice do mês de janeiro do ano de 2010, os valores apresentados serão o acúmulo de precipitação de julho a dezembro de 2009 em comparação ao histórico de precipitação do mesmo período nos anos anteriores. Assim, plotou-se o índice referente ao mês de julho de 2015 (Figura 2) dentro dos limites administrativos do estado do Pará.

Como citado, o índice indica quando a precipitação está elevada ou escassa dentro de um período de tempo. Ao plotar-se o SPP de julho de 2015, calcula-se a precipitação acumulada de janeiro a junho de 2015 comparada com a precipitação de janeiro a junho dos anos anteriores, e é notado que há umidade na região sul, porção noroeste e norte do estado, enquanto que a falta de umidade no comparativo está contida na região centro-oeste, porção noroeste e norte. 
Figura 2 - Distribuição espacial acumulada do SPI no estado do Pará para o período de 2005 a 2015, por avaliação semestral.
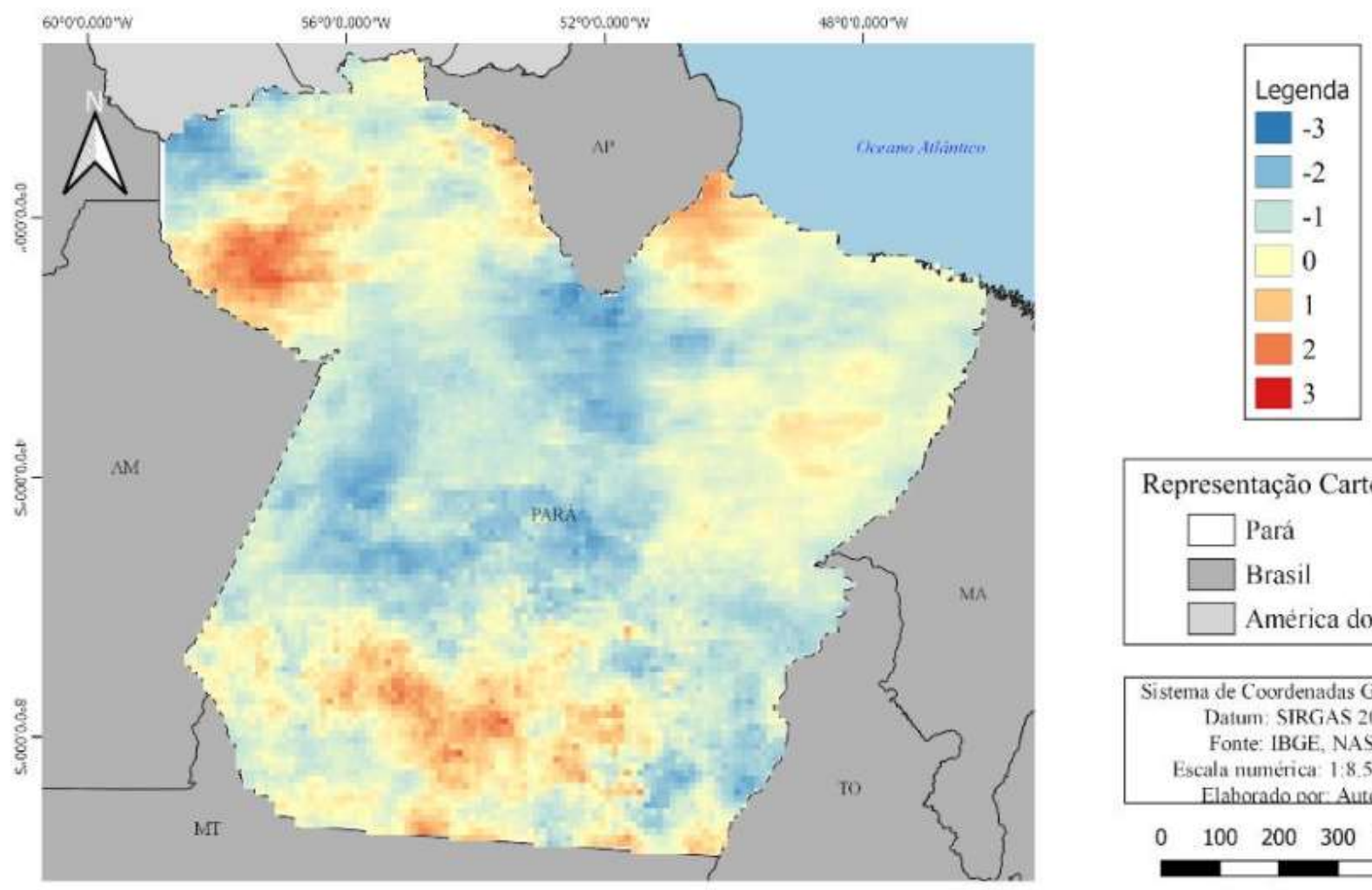

Fonte: NASA (2020)

Visando compreender melhor, buscou-se dados de precipitação mensais disponibilizados pelo Instituto Nacional de Meteorologia (INMET) no período deste trabalho vistos nas tabelas 2 e 3 para os municípios de Belém e Itaituba, com estações convencionais descritas com seu posicionamento na Tabela 1.

Tabela 1 - Coordenadas geográficas das estações.

\begin{tabular}{ccc}
\hline Estação (código) & Lat & Lon \\
\hline Belém (82191) & $-01^{\circ} 26^{\prime} 06^{\prime \prime}$ & $-48^{\circ} 26^{\prime} 16^{\prime \prime}$ \\
Itaituba (82445) & $-04^{\circ} 16^{\prime} 38^{\prime \prime}$ & $-55^{\circ} 59^{\prime} 35^{\prime \prime}$ \\
\hline
\end{tabular}

Fonte:Brasil (2009).

Foi verificado pelo software QGIS que na localização da estação de Belém, o IPP teve um valor de -0,9612. Como explicado, ainda não é considerado como uma baixa precipitação ou relacionado com a seca, este baixo valor pode ser entendido pelo fato de que em 2014 o acúmulo total de precipitação foi de 2752,4mm enquanto que o acúmulo em 2015 foi de 2692,6mm. O índice mostrou sensibilidade a essa variação apesar de o acúmulo de chuva nos anos anteriores não tenha sido de grande diferença. 
Tabela 2 - Quantidade de chuva por mês na estação Belém em milimetros.

\begin{tabular}{cccccccccccc}
\hline Mês & \multicolumn{10}{c}{ Anos } \\
\hline & 2005 & 2006 & 2007 & 2008 & 2009 & 2010 & 2011 & 2012 & 2013 & 2014 & 2015 \\
\hline Jan & 249,8 & 387,8 & 306,9 & 553,8 & 354,5 & 452,3 & 520,3 & 451,2 & 372,7 & 406,9 & 503,2 \\
Fev & 363,9 & 275,1 & 442,9 & 468,4 & 422,0 & 359,7 & 332,4 & 411,9 & 612,5 & 602,9 & 414,3 \\
Mar & 413,7 & 685,6 & 419,8 & 457,4 & 582,3 & 296,8 & 490,9 & 742,5 & 532,3 & 562,7 & 540,3 \\
Abr & 565,1 & 495,7 & 459,7 & 505,1 & 469,9 & 450,4 & 579,4 & 382,8 & 527 & 561,2 & 604,9 \\
Maio & 449,2 & 325,6 & 265,9 & 308,1 & 456,6 & 403,0 & 477,3 & 225,1 & 409,6 & 332,9 & 413,7 \\
Jun & 257,8 & 121 & 219,9 & 336,7 & 317,3 & 176,3 & 241,5 & 320 & 150,5 & 285,8 & 216,2 \\
\hline
\end{tabular}

Fonte: Adaptado de INMET (2020).

Na estação de Itaituba, o valor IPP se mostrou como uma boa quantidade negativa (- 2,3472). Olhando para os valores de precipitação correspondente a este município (Tabela 3), o acúmulo de chuva em 2014 foi de 1506,9mm, em 2013 foi de 1542,6mm, em 2012 foi de 1529,1mm e seguindo nessa linha até 2005, enquanto que o acúmulo em 2015foi de 1089,9mm, um tanto inferior aos anos anteriores.

Tabela 3 - Quantidade de chuva por mês na estação Itaituba em milimetros.

\begin{tabular}{lcccccccccccc}
\hline Mês & \multicolumn{10}{c}{ Anos } \\
\hline & 2005 & 2006 & 2007 & 2008 & 2009 & 2010 & 2011 & 2012 & 2013 & 2014 & 2015 \\
\hline Jan & 177,8 & 106,7 & 196,4 & 108,9 & 193,1 & 132,1 & 195,3 & 313,2 & 220,8 & 240,0 & 172,4 \\
Fev & 103,0 & 236,1 & 135,3 & 151,5 & 92,7 & 188,1 & 179,4 & 112,2 & 245,5 & 84,8 & 100,6 \\
Mar & 141,6 & 155,9 & 96,9 & 109,6 & 134,1 & 95,5 & 60,3 & 197,0 & 110,4 & 211,2 & 96,7 \\
Abr & 242,1 & 113,6 & 168,9 & 110,6 & 142,3 & 152,1 & 139,1 & 44,3 & 83,9 & 82,2 & 26,4 \\
Maio & 105,0 & 240,9 & 119,1 & 135,2 & 45,1 & 134,2 & 174,6 & 103,0 & 210,7 & 41,3 & 98,3 \\
Jun & 459,5 & 519,8 & 454,5 & 208,3 & 253,7 & 224,8 & 201,7 & 259,8 & 299,7 & 186,5 & 258,2 \\
\hline
\end{tabular}

Fonte: Adaptado de INMET (2020).

\section{Conclusão}

O Índice de Precipitação Padrão se mostrou adequado quando se buscou dados numéricos de fontes confiáveis. Porém vale lembrar que é um índice estatístico que leva em consideração somente os dados de precipitação, podendo ser influenciado por outras variáveis quanto ao seu resultado, haja visto que quanto menor o período do índice (um, três meses) mais sensível ele se mostra.

É uma ferramenta de boa confiabilidade quando aplicada corretamente, oferecendo cobertura de dados global e de maneira indireta, não necessitando de instrumentos em terra e somente da constelação de satélites artificiais voltados a este propósito, além do conhecimento e capacidade do processamento.

Sugere-se estudos de precipitação mais refinados quanto a verificabilidade dos resultados como um quantitativo maior de comparações de informações acerca da pluviometria, período de tempo maior de estudo e as diversas escalas de índice. 


\section{Referências}

Balbino, A. (2016). Estação meteorológica: como funciona e sua importância na agricultura. Agrosmart cultivo inteligente. https://agrosmart.com.br/blog/estacao-meteorologica-funciona-importancia-agricultura/.

Brasil. (2020). Instituto Nacional de Meteorologia. Disponível em: https://tempo.inmet.gov.br/Graficos/A001.

Brasil. (2009). Ministério do Meio Ambiente. Inventário Estações Pluviométricas. (2a ed.), ANA, SGH, 495p.

Collischonn, B., Collischonn, W., Silva, B. C., \& Tucci, C. E. M. (2016). Simulação hidrológica da bacia do rio São Francisco usando precipitação estimada pelo satélite TRMM: resultados preliminares. < http://rhama.net/download/artigos/artigo114.pdf >.

Embrapa. (2004). ABC da agricultura familiar. Formas de garantir água nas secas, vol.1. A chuva no sertão. 19p. https://ainfo.cnptia.embrapa.br/digital/bitstream/item/215833/1/A-chuva-no-sertao.pdf.

Grilli, M. (2021). Desmatamento e queimadas alteram ciclos de chuva e reduzem retenção de água no solo. Mudanças no uso da terra desregulam processo de evapotranspiração das plantas, afetando a disponibilidade hídrica. Um só planeta, Globo Rural Digital. https://revistagloborural.globo.com/Um-SoPlaneta/noticia/2021/03/desmatamento-e-queimadas-alteram-ciclos-de-chuva-e-reduzem-retencao-de-agua-no-solo.html.

Huffman, G. J., Adler, R. F., Bolvin, D. T., Gu, G., Nelkin, E. J., Bowman, K. P., Hong, Y., Stocker, E. F., \& Wolff, D. B. (2007). The TRMM multisatellite precipitation analysis: Quasi-global, multi-year, combined-sensor precipitation estimates at fine scale. Journal of Hydrometeorology, 8(1), 38- 55. https://dx.doi.org/10.1175/JHM560.1.

Instituto nacional de meteorologia-INMET. (2021). Sobre meteorologia. Previsão do tempo. https://portal.inmet.gov.br/sobre-meteorologia.

Mol, J. M. D. (2005). Estimativa de Precipitação por meio de Sensoriamento Remoto. Dissertação de Mestrado - Universidade de Brasília. Faculdade de Tecnologia. Departamento de Engenharia Civil e Ambiental. xv, 88 p. http://ptarh.unb.br/wp-content/uploads/2017/03/JulianaMaria.pdf

NASA - National Aeronautics and Space Administration. (2021). IMERG: Integrated Multi-satellitE Retrievals for GPM. https:/gpm.nasa.gov/data/imerg.

NASA - National Aeronautics and Space Administration.(2020). NASA's Applied Remote Sensing Training Program - ARSET. Applications of GPM IMERG reanalysis for assessing extreme dry and wet periods. https://appliedsciences.nasa.gov/join- mission/training/english/applications-gpm-imerg-reanalysisassessing-extreme-dry-and-wet.

National Drought Mitigation Center. (2020). Universidade de Nebraska. https://drought.unl.edu/droughtmonitoring/SPI/MapInterpretation.aspx.

Pinheiro, M. P. M. A., Ramires, T., \& Manzione, R. L. (2017). Análises estatísticas da similaridade entre dados agrometeorológicos de superfície e obtidos por sensores remotos orbitais. Anais do XVIII Simpósio Brasileiro de Sensoriamento Remoto-SBSR. $28-31$ de maio. file://C:/Users/franc/OneDrive/\%C3\%81rea\%20de\%20Trabalho/PONTOS\%2019-2021/Hidrologia/galoa-proceedings--sbsr--59240.pdf.

Santos, G.G., Griebeler, N.P. \& Oliveira, L.F.C. (2010). Chuvas intensas relacionadas à erosão hídrica. Revista Brasileira de Engenharia Agrícola e Ambiental $14(2), 115-123$

Silva, D. T., Bispo, B. R. S., Almeida, A. Q., Silva, R. M. \& Cruz, M. A. S. (2020). Precipitação estimada por sensoriamento remoto no estado de Sergipe. Nativa, 8(2), 10.31413/nativa.v8i2.7821

Siqueira, B., \& Nery, J. T. (2017). Análise do Índice Padronizado de Precipitação para o Estado de São Paulo. Revista brasileira de geografia física. 10(6). https://periodicos.ufpe.br/revistas/rbgfe/article/view/234110/27937.

Sparovek, G. (2014). Köppen's climate classification map for Brazil. Meteorologische Zeitschirift, 22(6), 711-728.

Tessari, P. M. B., Oliveira Júnior, J. F., Góis, G., \& Galvani, E. (2018). Variabilidade do Índice de Precipitação Padronizada na Região Norte do Estado do Paraná Associada aos Eventos El Niño - Oscilação Sul. Revista Brasileira de Meteorologia, 33(1).

U.S. Drought Portal. (2020). Data and Maps. https://www.drought.gov/drought/climate-and-drought-indices-python.

Vianello, R. L. (2011). A estação meteorológica e seu observador. Uma parceria secular de bons serviços prestados à humanidade. Instituto Nacional de Meteorologia. 19p. https://portal.inmet.gov.br/uploads/publicacoesDigitais/aestacaometeorologicaeseuobservador.pdf.

Wagner, P. D., Fiener, P., Wilken, F., Kumar, S., \& Schneider, K. (2012). Comparison and evaluation of spatial interpolation schemes for daily rainfall in data scarce regions. Journal Hydrometeorology, 464-465, 388-400, 10.1016/j.jhydrol.2012.07.026.

Zhong, L. (2012). Comparison of precipitation estimates between Version 7 3-hourly TRMM Multi-Satellite Precipitation Analysis (TMPA) near-real-time and research products. Atmospheric Research, 153, 119-133. http://dx.doi.org/10.1016/j.atmosres.2014.07.032. 